\title{
Greater Mindfulness is Linked to Less Procrastination
}

\section{Nicola S. Schutte ${ }^{1}$ (i) $\cdot$ Andrea del Pozo de Bolger ${ }^{1}$}

Accepted: 18 December 2019 / Published online: 2 January 2020

(C) Springer Nature Switzerland AG 2019

\begin{abstract}
This study examined the relationships between trait mindfulness, a positive psychological characteristic, attention and procrastination. One hundred and seventy-four Australian participants, 133 women and 41 men, with a mean age of 35.44, completed measures of trait mindfulness, ability for sustained attention and procrastination. Participants recorded a task they thought they might delay completing and were randomly assigned to a brief mindfulness exercise or a control exercise. Participants afterwards rated their intention to work on the task. Greater trait mindfulness was associated with better ability to sustain attention and with less procrastination. Attention mediated the relationship between mindfulness and procrastination. With engagement held constant, participants in the mindfulness exercise condition expressed more intention to work towards completing a task on which they tend to procrastinate than participants in the control condition. These findings have implications for better understanding procrastination and for the use of mindfulness to assist those who procrastinate.
\end{abstract}

Keywords Attention · Mindfulness $\cdot$ Procrastination

Procrastination consists of delaying a task for a maladaptively long time (Baumeister et al. 1994; Steel 2007). A substantial number of adults often procrastinate (Ferrari et al. 2005; Steel 2007) and harmful procrastination is especially prevelant among university students, with estimates of frequent procrastination in this group ranging from $50 \%-$ 95\% (Kim and Seo 2015; Steel 2007). Individuals who procrastinate tend to recognize that their procrastination is harmful, and most of those who procrastinate would like to change this behavior (Steel 2007). Procrastination is associated with poor performance (Kim and Seo 2015), experience of stress (Beutel et al. 2016; Sirois 2014), and depression and anxiety (Beutel et al. 2016; Klein et al. 2017).

Nicola S. Schutte

nschutte@une.edu.au

1 University of New England, Armidale, NSW 3351, Australia 
Several characteristics of those who tend to procrastinate provide insight into the nature of procrastination. In a meta-analytic examination combining the results of 10 studies, Steele (Steel 2007) found an association of $r=.40$ between procrastination and finding tasks in general aversive. Steele's (Steel 2007) other meta-analytic findings included an association of $r=.45$ between procrastination and distractibility across 13 studies and an association of $r=.58$ between procrastination and lower self-control across 21 studies.

Self-Regulation Theory (Baumeister et al. 1994) provides a theoretical foundation for understanding procrastination. This theory proposes that a motivational conflict stemming from disparity between internal standards and focus on more immediate pleasurable activities or aspects of task aversion lead to delaying tasks. This motivational conflict described by Self-Regulation Theory involves fracturing of attention between internal and external experiences and judgemental evaluation of the lure of approach towards pleasurable activities or avoidance of unpleasant tasks. Further, such conflicts and diversion of attention depletes an individual's resources, which may lead to delaying tasks.

The characteristic of mindfulness may be linked to procrastination, with greater mindfulness being associated with less tendency to procrastinate. Mindfulness consists of non-judgmental awareness and acceptance of experience (Kabat-Zinn 2005; Shapiro et al. 2006). Non-judgemental awareness may reduce the likelihood of motivational conflicts leading to procrastination as proposed by Self-Regulation Theory (Baumeister et al. 1994). Several studies have found associations between greater mindfulness and less procrastination (Cheung and Ng 2019; Glick et al. 2014; Howell and Buro 2011; Sirois and Tosti 2012). For example, Cheung and Ng (2019) found concurrent associations between greater mindfulness and less procrastination, with a correlation of -.41 at an initial measurement, and further found that initial mindfulness longitudinally predicted less procrastination at later measurement times, with initial procrastination held constant.

Baer et al. 2008provided empirically-based detail relating to the dimensions of nonjudgmental awareness and acceptance that define mindfulness. They identified the following five mindfulness facets: observing, noticing, or attending to thoughts, feelings, perceptions, or sensations; describing or labelling these with words; acting with awareness; being nonreactive to inner experience; and, not judging experience. Of these facets, not judging and non- reactive awareness may be the most important (Emerson et al. 2017; Medvedev et al. 2018). In neuro-cognitive examinations of mindfulness, Vago and Sibersweig (2012) proposed that self-awareness encompassing ability to selfregulate is central to mindfulness. Wheeler et al. 2017reviewed neurological evidence suggesting that attention and regulation of emotion are central to mindfulness.

As well as being a trait, or habitual pattern of perception and thought, mindfulness can be a momentary state (Kiken et al. 2015). Trait mindfulness may give rise to more mindful states and conversely, frequent mindful states may build trait mindfulness (Kiken et al. 2015). An aspect of a mindful state is awareness and attention to the moment (Kabat-Zinn 2005). Increases in mindfulness may lead to changes in cognition, including better ability to sustain attention. For example, Zeidan et al. (2010) found that brief mindfulness training resulted in sustained attention as assessed by performance tests. Wong et al. (2018) found that mindfulness training led to improved sustained attention assessed by a performance test and by EEG. Engagement, or active 
involvement, can facilitate acquisition of skills (Reeve 1996). Greater engagement with the learning of mindfulness skills may help encode these skills.

The non-judgmental aspect of mindfulness may make it less likely that an individual high in mindfulness will judge tasks as aversive, which is characteristic of procrastination. Awareness, attention, non-reactivity and self-regulation aspects of mindfulness may make it less likely that an individual high in mindfulness will have low self-control or will be distracted, characteristics associated with procrastination. Some support for these propositions come from Glick et al. (2014) and Sirois and Tosti (2012), with both studies finding significant associations between the higher scores on the awareness aspect of mindfulness as well as the non-judgement aspect of mindfulness with less procrastination.

Attention and awareness are central aspects of mindfulness. Symptoms relating to inattention are characteristic of attention-deficit hyperactivity disorder (ADHD) (American Psychiatric Association 2013). Mindfulness training has shown some success in improving these symptoms in individuals with ADHD (Househam and Solanto 2016; Hoxhaj et al. 2018; Mitchell et al. 2017; Van de Weijer-Bergsma et al. 2012), suggesting that mindfulness may improve attention among individuals with deficits in this quality. Likewise, a path between greater mindfulness and less procrastination may be the ability to sustain attention facilitated by mindfulness.

Mindfulness is a concept distinct from the perceptions of task aversion, distractibility and self-control that have been found to be associated with procrastination (Steel 2007); however, certain features of mindfulness may make it less likely that an individual high in mindfulness will judge tasks to be aversive, be distracted, or have low self-control. In relation to the Self-Regulation Theory foundation of procrastination, mindfulness may make is less likely that attention is fractured or that temptation to deviate from internal standards is judged harshly by the self. Thus, mindfulness may help an individual conserve resources, and such conservation of resources may reduce the likelihood of procrastination.

Procrastination may be changeable. Intervention studies drawing on approaches such as cognitive behavior therapy (e.g., Eckert, 2018; Rozental, Forsell, Svensson, Andersson, \& Carlbring, 2015) have shown some promise in reducing procrastination. In a review, Zacks and Hen (2018) concluded that there have been relatively few procrastination intervention studies because of the numerous possible underlying factors contributing to procrastination. Low mindfulness may be one underlying factor, and to date no systematic studies have explored mindfulness as an intervention strategy for assisting those who procrastinate. Based on observations of procrastination among students who had sought assistance from writing tutors, Kervin and Barrett (2018) suggested that judgement of the self may lead to negative emotions, which students may deal with through procrastination on tasks which lead to negative evaluations of the self. They proposed that facilitation of mindfulness may assist in helping students overcome procrastination tendencies by increasing non-reactive selfawareness and lessening judgement of the self, which in turn would lessen the propensity to procrastinate.

The aim of the present study was to examine the relationships between trait mindfulness, attention, and procrastination. Model testing assessed the possible mediating role of attention in the relationship between mindfulness and procrastination. An experimental pilot study explored the potential causal role of mindfulness in procrastination through a brief state-level mindfulness intervention. 
The hypotheses were as follows:

1. Greater mindfulness is associated with better ability to sustain attention.

2. Greater mindfulness is associated with less procrastination.

3. Better ability to sustain attention is associated with less procrastination.

4. Ability to sustain attention is a path connecting greater mindfulness with less procrastination (i.e., focus mediates between mindfulness and procrastination).

5. Participants who engage in a brief mindfulness exercise will afterwards have more intention to work towards completing a task on which they tend to procrastinate than those in a control condition.

\section{Methods}

\subsection{Participants and Procedure}

Participants were recruited after approval from the university institutional review board and all participants provided informed consent. The 174 participants included 133 women and 41 men, with 170 participants completing all measures. Four participants discontinued participation while completing the pre-measures. Participants were both mature age and traditional age university students from Australia, with a mean age of 35.44, $S D=11.46$. With power set at .80 , this number of participants is adequate to detect a medium effect size in a mediation analysis and is adequate to detect a medium effect size in a two group comparison. Please see Fig. 1 describing the participant flow through the study.

After approval of the study by the university institutional review board, participants provided informed consent and completed measures of trait mindfulness, attention and procrastination on the Qualtrics electronic research platform. Participants also recorded a task they would like to complete in the next month that they thought they might delay completing. To help ensure completion of measures, the research platform was programmed to only allow progression through the study when participants provided responses to items. After completing pre-measures, participants were randomly assigned, with equal numbers to conditions, through the on-line platform, to either a brief three minute mindfulness exercise consisting of audio instructions intended to induce non-judgmental awareness and attention to present body sensations, including breath (Penman 2011), or a control exercise consisting of the instruction to reflect on activities of the previous day and to make brief written notes of these activities. In relation to the mindful state induction condition, non-evaluative focus on body sensations, with a special emphasis on breath, is a core aspect of a number of successful mindfulness inducing mediation techniques (Burke 2012). The mindfulness exercise, practiced once, was intended to facilitate a mindful state. An equal amount of time, three minutes, was allotted to each condition. Participants in both conditions rated their engagement with the activity comprising their respective exercises immediately after completing the exercises. Finally, immediately after completion of the relevant condition, participants in both conditions rated their intention to work on the task they had earlier indicated they would like to complete in the next month. 
Fig. 1 Flow of Participants through Study

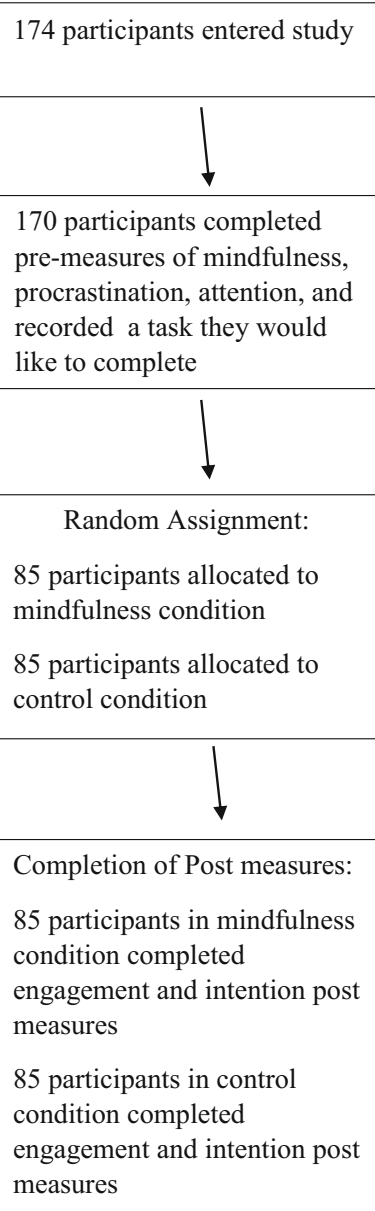

\section{Measures}

\subsection{Mindfulness}

The short form of the Freiburg Mindfulness Inventory (Kohls et al. 2009; Walach et al. 2006) assessed trait mindfulness, conceptualized as comprising awareness and acceptance, or non-judgement. Respondents rate themselves on a 5-point scale on items such as "I am open to the experience of the present moment." and "I see my mistakes and difficulties without judging them." Previous research has shown the measure to have good internal consistency and evidence of validity (Walach et al. 2006), with community participants with and without meditation experience (Kohls et al. 2009). In the present study internal consistency, assessed by Cronbach's alpha, was .88. 


\subsection{Procrastination}

Lay's General Procrastination Scale (Lay 1986) assessed global tendencies towards procrastination. Respondents rate themselves on a 5-point scale on items such as "I often find myself performing tasks that I had intended to do days before. "and "In preparing for some deadline, I often waste time by doing other things. "Previous research indicates that the measure has good internal consistency of .82, and evidence of validity (Lay 1986). In the present study internal consistency, assessed by Cronbach's alpha, was .88.

\subsection{Attention}

The Adult ADHD Self-Report Scale-V1.1 Symptoms Checklist (ASRS-V1.1), which provides a continuous score of typical sustained attention versus inattention, assessed participants' general ability for sustained attention. Respondents rate themselves on a 5point scale on items such as "How often do you have difficulty keeping your attention when you are doing boring or repetitive work?" and "How often do you have difficulty concentrating on what people say to you, even when they are speaking to you directly?". Attention was coded so that higher scores indicated better attention. Previous research indicates that this measure has good internal consistency, with Cronbach's alpha of .88, and evidence of validity (Adler et al. 2006). The scale has been used with various populations to assess ability for sustained attention on a continuum, including with university student samples (Gropper and Tannock 2009). In the present study internal consistency, assessed by Cronbach's alpha, was .77.

\subsection{Intention towards Task}

Before being randomly assigned to either a mindfulness or control exercise, participants recorded a task they would like to complete in the next month that they thought they might delay completing. After participation in the experimental or control exercise, participants then saw the task they recorded piped in as the stem for their ratings of items assessing intention towards completing the task in the next month. For example, if a participant recorded 'Paint House' prior to random assignment to a condition, the post-condition intention assessment stem read 'Previously you described a task you would like to complete in the next month. This task is Paint House. Please rate what you are likely to do regarding this task on the following statements.' Participants then rated their agreement with the following three statements using a seven-point scale: 'I will work hard on completing this task', 'I will wait to start working towards this task' (reversed), 'I will start working towards this task today'. The internal consistency of this three-item intention measure, assessed by Cronbach's alpha, was .74.

\subsection{Engagement}

Following the experimental and control condition exercises, participants were asked to indicate on a scale from 0 (not at all) to 100 (a great deal) to their level of engagement with the exercise. 


\section{Results}

\subsection{Demographic Differences}

There were no significant differences between women and men in mindfulness, attention or procrastination (see Table 1). Older participants were more likely to have sustained attention and were less likely to procrastinate (see Table 1). Age was not significantly associated with mindfulness (see Table 1).

\subsection{Associations between Mindfulness, Attention and Procrastination}

Pearson $r$ correlations tested the hypotheses that greater mindfulness is associated with better ability to sustain attention, that greater mindfulness is associated with less procrastination, and that better ability to sustain attention is associated with less procrastination were tested with. (see Table 1). Greater mindfulness was significantly associated with better ability to sustain attention and was significantly associated with less procrastination. Better ability to sustain attention was significantly associated with less procrastination. Because older age was associated with greater attention and less procrastination, partial correlations investigated whether the associations between mindfulness, attention and procrastination would still be significant when age was held constant. The partial correlation results showed the relationships to still be significant and of similar magnitude when age was held constant.

\subsection{The Relationship between Mindfulness and Procrastination Mediated by Attention}

The hypothesis that ability to sustain attention is a path connecting greater mindfulness with less procrastination was tested through mediation analysis using an SPSS macro, PROCESS model 4 . The model was significant, $\mathrm{R}=.60, p=.001$. Ability for sustained attention significantly mediated the relationship between mindfulness and procrastination. Please see Fig. 2 for the mediation model and results.

Table 1 Descriptive statistics and correlations

\begin{tabular}{lllllll}
\hline Variable & Gender & Age & Mindfulness & Attention & Procrastination \\
\hline 1. & Gender & - & & & & \\
2. & Age & .03 & - & & & \\
3. & Mindfulness & -.08 & .15 & - & & \\
4. & Attention & .01 & $.27^{* *}$ & $.38^{* *}$ & - & \\
5. & Procrastination & -.02 & $-.24^{* *}$ & $-.27^{* *}$ & $-.56^{* *}$ & - \\
& $M$ & - & 35.44 & 34.05 & 49.92 & 58.04 \\
& $S D$ & - & 11.46 & 7.44 & 7.29 & 23.32 \\
\hline
\end{tabular}

$n=170 . * * p<.01 .{ }^{\text {a }}$ Dummy coded $($ Male $=1 ;$ Female $=2)$ 


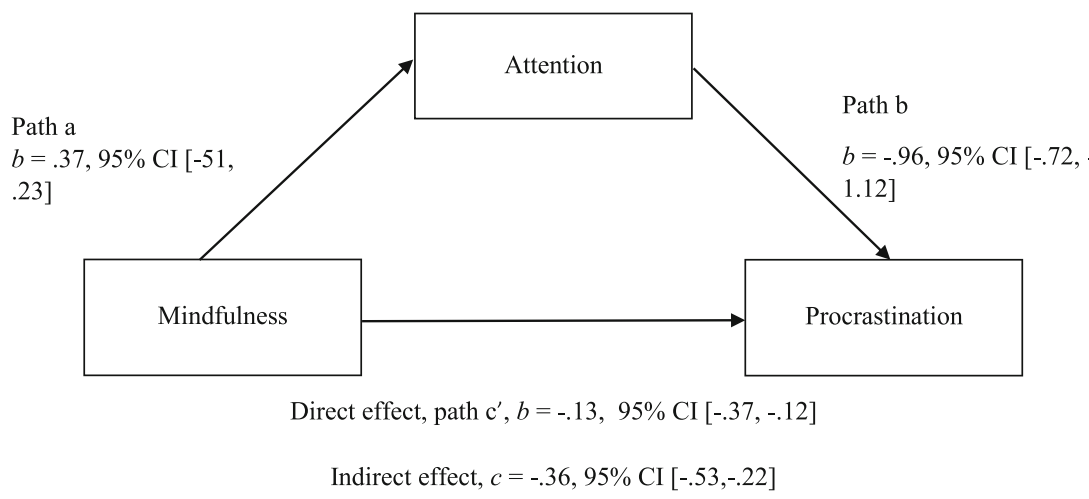

Fig. 2 Model and Mediation Results of Mindfulness as a Predictor of Procrastination, mediated by Attention, with the indirect effect based on 2000 bootstrap resamples. Values are unstandardised beta coefficients. $N=$ 170

\subsection{The Effect of a Brief Mindfulness Exercise on Intention to Work on Task}

Analysis of Covariance (ANCOVA) tested the hypothesis that the participants who engaged in a brief mindfulness exercise would afterwards report more intention to work towards completing a task on which they tended to procrastinate. Engagement with the intervention exercise was associated with intention to work on the task, $r(169)=.23, p=.01$, and this was used as the covariate in the analysis, with condition as the independent variable and intention to work on the task as the dependent variable. Engagement was selected as a covariate both because of its relevance to the impact of the mindfulness intervention and because of its relationship with intention to work on the task. The ANCOVA showed that the 85 participants in the mindfulness condition had a greater intention to work on the task (mean $=13.82, S D=3.98)$ than the 85 participants in the control condition (mean $=12.92, S D=4.09), F(2,167)=5.45$, $p=.02$, two-tailed. As older age was associated with less tendency to procrastinate in the pre-measures, another ANCOVA used both engagement and age as covariates. This analysis also showed that the 85 participants in the mindfulness condition had a greater intention to work on the task than the 85 participants in the control condition, $F(2,166)=5.58, p=.02$, two-tailed.

When engagement and age were not held constant, there was no two-tailed significant difference between groups, $F(1,168)=2.19, p=.14$, two-tailed.

\section{Discussion}

The results of the study supported the hypotheses that trait mindfulness is associated with better ability to sustain attention and less tendency to procrastinate. The results also supported the hypothesis that better ability to sustain attention is associated with less procrastination and that ability for sustained attention links greater trait mindfulness with less tendency to procrastinate. These findings add to the body of literature demonstrating the utility of mindfulness. 
The finding of the present study that greater mindfulness is associated with less tendency to procrastinate corroborates Glick et al.'s (2014) and Sirois and Tosti's (2012) findings regarding the relationship between mindfulness and procrastination. The findings of the present study also are congruent with previous findings regarding mindfulness and sustained attention (Wong et al. 2018; Zeidan et al. 2010). The present study extends these previous findings in suggesting that the ability to sustain attention facilitated by mindfulness may link greater mindfulness with less procrastination.

To explore both the possible causal link between mindfulness and procrastination in a small pilot test, the present study randomly assigned participants to a brief mindfulness exercise or a control exercise. When engagement, as reported by participants, with the exercise was held constant, participants in the mindfulness exercise condition reported significantly more intention to work on a task on which they might procrastinate than participants in the control condition; when engagement was not held constant, the difference between the conditions was not significant.

These findings from the pilot experimental portion of the study provide tentative support for a causal role of mindfulness in reducing procrastination. The pilot study intervention was focused on participants' state of mindfulness. Repeated mindfulness states may result in increases in trait mindfulness (Kiken et al. 2015). Future research might examine more comprehensive mindfulness training intended to increase trait mindfulness and assess the effects of this on general tendencies to procrastinate.

Comprehensive mindfulness training to lessen procrastination might be based on Mindfulness Based Stress Reduction (MBSR, Kabat-Zinn 2013). Mindfulness Based Stress Reduction programs encompass a series of exercises, such as mindfulness meditation, and preparation for applying mindfulness in everyday situations, that are intended to develop mindfulness capacity (Grossman et al. 2004). Meta-analyses of the effect of mindfulness-based interventions have found a significant benefit of mindfulness training on both mental health outcomes and physical health outcomes across studies (Grossman et al. 2004; Khoury et al. 2013). A randomized controlled trial of MBSR intended to increase trait mindfulness in order to assist those who procrastinate might find that lessening of procrastination adds to the list of beneficial outcomes resulting from MBSR. Such research might help determine the optimal length and format of mindfulness interventions intended to assist those who procrastinate and might explore approaches to increasing engagement with mindfulness exercises, possibly through innovative technologies, such as use of virtual reality.

The results of the present study should be interpreted in light of several limitations. First, the model-testing portion of the study examining the relationships between mindfulness, sustained attention and procrastination was based on a concurrent data collection design and does not allow conclusions regarding causality. The suggestive findings from the small random assignment experimental pilot study showed that the state-based mindfulness exercise resulted in significantly less tendency to procrastinate on a task compared to the control exercise, but only when engagement with the exercise as reported by participants was held constant. It may be that an adequate level of engagement is needed in order for brief mindfulness interventions to have an effect. Second, participants were university students from one country. The results might not generalize to other populations. Future research might investigate the applicability of the findings for other populations. 
Future research might build on Self-Regulation Theory (Baumeister et al. 1994) applications in other areas in conjunction with mindfulness training to develop the most effective procrastination intervention programs. For example, such research might investigate whether mindfulness training buffers exhaustion of self-regulation capacity in relation to procrastination under demanding circumstances. Future research might also investigate the impact of mindfulness training in preventing procrastination in specific realms of life.

In conclusion, the results of the present study suggest that greater trait mindfulness is linked to less procrastination, and that this relationship is mediated by the sustained attention facilitated by mindfulness. Mindfulness-based programs intended to increase trait mindfulness through repeated inductions of state mindfulness may have promise in assisting those who procrastinate.

Funding There is no funding source.

\section{Compliance with Ethical Standards}

Conflict of Interest The authors declare that they have no conflict of interest.

Ethical Approval The research was approved by the institutional ethics committee and was performed in accordance with the ethical standards as laid down in the Declaration of Helsinki.

Informed Consent Informed consent was obtained from all individual participants included in the study.

\section{References}

Adler, L. A., Spencer, T., Faraone, S. V., Kessler, R. C., Howes, M. J., Biederman, J., \& Secnik, K. (2006). Validity of pilot adult ADHD self-report scale (ASRS) to rate adult ADHD symptoms. Annals of Clinical Psychiatry, 18, 145-148. https://doi.org/10.1080/10401230600801077.

American Psychiatric Association. (2013). Diagnostic and statistical manual of mental disorders (5th ed.). Washington, DC: American Psychiatric Association.

Baer, R. A., Smith, G., Lykins, E. L. B., Button, D., Krietemeyer, J., Sauer, S., et al. (2008). Construct validity of the five facet mindfulness questionnaire in meditating and non-meditating samples. Assessment, 15, 329-342. https://doi.org/10.1177/1073191107313003.

Baumeister, R. F., Heatherton, T. F., \& Tice, D. M. (1994). Losing control: How and why people fail at selfregulation. San Diego: Academic Press.

Beutel, M. E., Klein, E. M., Aufenanger, S., Brähler, E., Dreier, M., Müller, K. W., et al. (2016). Procrastination, distress and life satisfaction across the age range-a German representative community study. PloS One, 11, e0148054. https://doi.org/10.1371/journal.pone.0148054.

Burke, A. (2012). Comparing individual preferences for four meditation techniques: Zen, Vipassana (mindfulness), qigong, and mantra. Explore: The Journal of Science and Healing, 8, 237-242. https://doi. org/10.1016/j.explore.2012.04.003.

Cheung, R. Y., \& Ng, M. C. (2019). Being in the moment later? Testing the inverse relation between mindfulness and procrastination. Personality and Individual Differences, 141, 123-126. https://doi. org/10.1016/j.paid.2018.12.015.

Eckert, M., Ebert, D. D., Lehr, D., Sieland, B., \& Berking, M. (2018). Does sms-support make a difference? effectiveness of a two-week online-training to overcome procrastination. a randomized controlled Trial. Frontiers in Psychology, 9, Article 1103.

Emerson, L. M., Heapy, C., \& Garcia-Soriano, G. (2017). Which facets of mindfulness protect individuals from the negative experiences of obsessive intrusive thoughts?. Mindfulness, 1-11. Early view at https://doi.org/10.1007/s12671-017-0854-3. 
Ferrari, J. R., O'Callaghan, J., \& Newbegin, I. (2005). Prevalence of procrastination in the United States, United Kingdom, and Australia: Arousal and avoidance delays among adults. North American Journal of Psychology, 7, 1-6. Retrieved fromhttps://www.researchgate.net/profile/Joseph_Ferrari3 /publication/276354186_Prevalence_of_Procrastination_in_the_United_States_United_Kingdom_and_ Australia_Arousal_and_Avoidance_Delays_among_Adults/links/5557a6c108aeaaff3bf771 5 .pdff.

Glick, D. M., Millstein, D. J., \& Orsillo, S. M. (2014). A preliminary investigation of the role of psychological inflexibility in academic procrastination. Journal of Contextual Behavioral Science, 3, 81-88. https://doi. org/10.1016/j.jcbs.2014.04.002.

Gropper, R. J., \& Tannock, R. (2009). A pilot study of working memory and academic achievement in college students with ADHD. Journal of Attention Disorders, 12, 574-581. https://doi.org/10.1177 $/ 1087054708320390$.

Grossman, P., Niemann, L., Schmidt, S., \& Walach, H. (2004). Mindfulness-based stress reduction and health benefits: A meta-analysis. Journal of Psychosomatic Research, 57, 35-43. https://doi.org/10.1016/S00223999(03)00573-7.

Househam, A., \& Solanto, M. (2016). Mindfulness as an intervention for ADHD. The ADHD Report, 24, 1-9, 13.

Howell, A. J., \& Buro, K. (2011). Relations among mindfulness, achievement-related self-regulation, and achievement emotions. Journal of Happiness Studies, 12, 1007-1022. https://doi.org/10.1007/s10902010-9241-7.

Hoxhaj, E., Sadohara, C., Borel, P., D’Amelio, R., Sobanski, E., Müller, H., ... \& Philipsen, A. (2018). Mindfulness vs psychoeducation in adult ADHD: A randomized controlled trial. European Archives of Psychiatry and Clinical Neuroscience, 1-15. Early view at https://doi.org/10.1007/s00406-018-0868-4.

Kabat-Zinn, J. (2005). Coming to our senses: Healing ourselves and the world through mindfulness. New York: Hyperion.

Kabat-Zinn, J. (2013). Full catastrophe living, revised edition: How to cope with stress, pain and illness using mindfulness Meditation. Hachette.

Kervin, C. E., \& Barrett, H. E. (2018). Emotional management over time management: Using mindfulness to address student procrastination. WLN: A Journal of Writing Center Scholarship, 42, 10-18. Retrieved June 2019 from https:/go.galegroup.com/ps/anonymous?p=AONE\&sw=w\&issn=\&v=2.1\&it=r\&id= GALE\%7CA538859614\&sid=googleScholar\&linkaccess=fulltext.

Khoury, B., Lecomte, T., Fortin, G., Masse, M., Therien, P., Bouchard, V., \& Hofmann, S. G. (2013). Mindfulness-based therapy: A comprehensive meta-analysis. Clinical Psychology Review, 33, 763-771. https://doi.org/10.1016/j.cpr.2013.05.005.

Kiken, L. G., Garland, E. L., Bluth, K., Palsson, O. S., \& Gaylord, S. A. (2015). From a state to a trait: Trajectories of state mindfulness in meditation during intervention predict changes in trait mindfulness. Personality and Individual Differences, 81, 41-46. https://doi.org/10.1016/j.paid.2014.12.044.

Kim, K. R., \& Seo, E. H. (2015). The relationship between procrastination and academic performance: A meta-analysis. Personality and Individual Differences, 82, 26-33. https://doi.org/10.1016/j. paid.2015.02.038.

Klein, E. M., Beutel, M. E., Müller, K. W., Wölfling, K., Brähler, E., \& Zenger, M. (2017). Assessing procrastination: Dimensionality and measurement invariance of the general procrastination scale Screening (GPS-S) in a representative sample. European Journal of Psychological Assessment. Advance online publication. Ealy view at https://doi.org/10.1027/1015-5759/a000441.

Kohls, S., Sauer, S., \& Walach, H. (2009). Facets of mindfulness: Results of an online study investigating the Freiburg mindfulness inventory. Personality and Individual Differences, 46, 224-230. https://doi. org/10.1016/j.paid.2008.10.009.

Lay, C. H. (1986). At last, my research article on procrastination. Journal of Research in Personality, 20,474 495. https://doi.org/10.1016/0092-6566(86)90127-3.

Medvedev, O. N., Norden, P. A., Krägeloh, C. U., \& Siegert, R. J. (2018). Investigating unique contributions of dispositional mindfulness facets to depression, anxiety, and stress in general and student populations. Mindfulness, 1-11. Early view at doi.org/10.1007.

Mitchell, J. T., McIntyre, E. M., English, J. S., Dennis, M. F., Beckham, J. C., \& Kollins, S. H. (2017). A pilot trial of mindfulness meditation training for ADHD in adulthood: Impact on core symptoms, executive functioning, and emotion dysregulation. Journal of Attention Disorders, 21, 1105-1120. https://doi. org/10.1177/1087054713513328.

Penman, D. (2011). Mindfulness: Finding Peace in a Frantic World. Retrieved 1 Nov. 2017 from $\mathrm{http}: / /$ franticworld.com/the-three-minute-breathing-space-meditation-is-now-free-to-download/

Reeve, J. (1996). Motivating others: Nurturing inner motivational resources. Needham Heights: Allyn \& Bacon. 
Rozental, A., Forsell, E., Svensson, A., Andersson, G., \& Carlbring, P. (2015). Internet-based cognitivebehavior therapy for procrastination: A randomized controlled trial. Journal of Consulting and Clinical Psychology, 83, 808-824.

Shapiro, S. L., Carlson, L. E., Astin, J. A., \& Freedman, B. (2006). Mechanisms of mindfulness. Journal of Clinical Psychology, 62, 373-386. https://doi.org/10.1002/jclp.20237.

Sirois, F. M. (2014). Procrastination and stress: Exploring the role of self-compassion. Self and Identity, 13, 128-145. https://doi.org/10.1080/15298868.2013.763404.

Sirois, F. M., \& Tosti, N. (2012). Lost in the moment? An investigation of procrastination, mindfulness, and well-being. Journal of Rational-Emotive \& Cognitive-Behavior Therapy, 30, 237-248. https://doi. org/10.1007/s10942-012-0151-y.

Steel, P. (2007). The nature of procrastination: A meta-analytic and theoretical review of quintessential selfregulatory failure. Psychological Bulletin, 133, 65-94. https://doi.org/10.1037/0033-2909.133.1.65.

Vago, D. R., \& Sibersweig, D. A. (2012). Self-awareness, self-regulation, and self-transcendence (S-ART): A framework for understanding the neurobiological mechanisms of mindfulness. Frontiers in Human Neuroscience, 6, 1-30. https://doi.org/10.3389/fnhum.2012.0029.

Van de Weijer-Bergsma, E., Formsma, A. R., de Bruin, E. I., \& Bögels, S. M. (2012). The effectiveness of mindfulness training on behavioral problems and attentional functioning in adolescents with ADHD. Journal of Child and Family Studies, 21, 775-787. https://doi.org/10.1007/s10826-011-9531-7.

Walach, H., Buchheld, N., Buttenmuller, V., Kleinknecht, N., \& Schmidt, S. (2006). Measuring mindfulness The Freiburg mindfulness inventory (FMI). Personality and Individual Differences, 40, 1543-1555. https://doi.org/10.1016/j.paid.2005.11.025.

Wheeler, M. S., Arnkoff, D. B., \& Glass, C. R. (2017). The neuroscience of mindfulness: How mindfulness alters the brain and facilitates emotion regulation. Mindfulness, 8, 1471-1487. https://doi.org/10.1007 /s12671-017-0742-x.

Wong, K. F., Teng, J., Chee, M. W., Doshi, K., \& Lim, J. (2018). Positive effects of mindfulness-based training on energy maintenance and the EEG correlates of sustained attention in a cohort of nurses. Frontiers in Human Neuroscience, 12, 80. Early view at doi.org/10.3389/fnhum.2018.00080.

Zacks, S., \& Hen, M. (2018). Academic interventions for academic procrastination: A review of the literature. Journal of Prevention \& Intervention in the Community, 46, 117-130. https://doi.org/10.1080 $/ 10852352.2016 .1198154$.

Zeidan, F., Johnson, S. K., Diamond, B. J., David, Z., \& Goolkasian, P. (2010). Mindfulness meditation improves cognition: Evidence of brief mental training. Consciousness and Cognition, 19, 597-605. https://doi.org/10.1016/j.concog.2010.03.014.

Publisher's Note Springer Nature remains neutral with regard to jurisdictional claims in published maps and institutional affiliations. 